\title{
Aquiescência em autorrelatos: Introdução ao conceito, correlatos e possiveis soluções
}

\author{
Grazielli Padilha Vieira \\ Daniely Fernandes Kamazaki \\ Cristiano Weiss \\ Cristian Zanon \\ Denise Ruschel Bandeira
}

\section{RESUMO}

O estilo de resposta aquiescente é um padrão de resposta há muito reconhecido na literatura sobre psicometria e avaliação psicológica, assim como a tendência à utilização de pontos extremos e também a evitação de pontos extremos. A aquiescência pode ser definida como a tendência de concordar com itens de um questionário a despeito do conteúdo apresentado, especialmente em instrumentos que utilizam escalas do tipo Likert. Assim, o respondente pode endossar igualmente itens opostos (e.g., sou otimista; sou pessimista), afetando as evidências de validade e fidedignidade dos instrumentos. 0 objetivo deste artigo é fornecer uma introdução ampla do conceito, apresentando as principais abordagens explicativas, evidências atuais de correlatos e uma breve retomada histórica sobre o conceito. São apresentadas as clássicas abordagens de cunho motivacional e cognitivo e a recente proposta de modelos mais integrativos para o entendimento da aquiescência. Por fım, são apresentados e discutidos os dois modelos estatísticos mais utilizados para o controle da aquiescência em pesquisas com escalas do tipo Likert na área de desenvolvimento de testes e avaliação psicológica.

Palavras-chave: vieses de resposta; estilo de resposta; psicometria, avaliação psicológica.

\begin{abstract}
Acquiescence in self-reports: Introduction to the concept, correlates and possible solutions

The acquiescent response style is a response pattern that has long been recognized in the literature on psychometry and psychological assessment, as well as the tendency to use extreme points and also to avoid extreme points. Acquiescent response style consists of the tendency to agree with items of a questionnaire despite their content, especially on instruments that use Likert-type scales. Thus, respondents may endorse opposed items equally (e.g., I am optimistic; I am pessimistic), affecting the instrument's validity evidence and reliability. This article aims to provide a broad introduction to the concept, presenting the main explanatory approaches, current evidence of correlates, and a brief historical review of the concept. We present classic motivational and cognitive approaches, as well as the recent proposal for more integrative models for understanding acquiescence. Finally, we present and discuss the two most used statistical models for the control of acquiescence in research with Likert-type scales in the area of test development and psychological assessment.
\end{abstract}

Keywords: Response bias; Response style; Psychometric, Psychological assessment.

Um recurso comumente utilizado no universo da avaliação psicológica e da pesquisa em ciências humanas e sociais são os questionários de autorrelato. Muitos deles utilizam escalas de tipo Likert, em que o respondente é orientado a marcar, em uma escala crescente de respostas, o quanto concorda ou discorda de um item (Edmondson, 2005). De forma

\section{Sobre os autores}

G. P. V.

http://orcid.org/0000-00016463-2443

Universidade Federal do Rio Grande do Sul - Porto Alegre, RS graziellipadilhavieira@gmail.com

D. F. K.

http://orcid.org/0000-00021556-7451

Universidade Federal do Rio Grande do Sul - Porto Alegre, RS kamazaki.psi@gmail.com

C. W. http://orcid.org/000000027017-8598

Universidade Federal do Rio Grande do Sul - Porto Alegre, RS cristianoweiss.psi@gmail.com

C. Z.

http://orcid.org/0000-00033822-5275

Universidade Federal do Rio Grande do Sul - Porto Alegre, RS crstn.zan@gmail.com

D. R. B.

http://orcid.org/0000-0001-

9867-2718

Universidade Federal do Rio

Grande do Sul - Porto Alegre, RS

bandeira@ufrgs.br

\section{Direitos Autorais}

Este é um artigo de acesso aberto e pode ser reproduzido livremente, distribuído, transmitido ou modificado, por qualquer pessoa desde que usado sem fins comerciais. 0 trabalho é disponibilizado sob a licença Creative Commons CC-BY-NC.

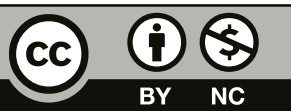




\section{M." INTERACÃO EM PSICOLOGIA}

geral, tem-se à disposição um leque contínuo de alternativas, como, por exemplo, "nem um pouco", "um pouco", "moderadamente", "bastante" e "extremamente". Assim, avalia-se a intensidade de identificação com as afirmações ou palavras apresentadas, as quais expressam características de um dado construto (Joshi et al., 2015). Teoricamente, pela qualidade gradual das opções oferecidas, as respostas tendem a representar os traços latentes dos indivíduos de forma mais apurada do que em um teste ou escala com opções binárias de resposta (sim ou não).

Embora muito popular por seus benefícios, esse formato de instrumento também pode apresentar limitações importantes (Ziegler, 2015). Há mais de meio século, observa-se a existência de certos padrões de resposta nos instrumentos que envolvem escalas, especialmente as do tipo Likert (Cronbach, 1946, 1950; Jackson \& Messick, 1958). Esses padrões não representam as características individuais buscadas pelo questionário, mas revelam um modo sistemático de responder aos itens de uma escala. Assim, estes podem ser entendidos como vieses de resposta e acabam por não refletir a real identificação do indivíduo nos construtos de interesse (Paulhus, 1991; Ziegler, 2015).

0 viés de resposta, como indica Ziegler (2015), refere-se a um termo guarda-chuva que envolve diferentes fenômenos. Dentro da nomenclatura utilizada na literatura internacional, encontram-se os conjuntos de resposta (response sets) e os estilos de resposta (response styles). 0 primeiro estaria ligado a fatores situacionais (e.g., pressão em função do tempo), caracterizando-se como uma reação temporária para uma demanda específica. Também é capaz de eliciar esse tipo de viés a própria apresentação do instrumento, como a construção ou a ordem dos itens no questionário (para uma revisão, ver Tourangeau \& Rasinski, 1988). Os estilos de resposta, por outro lado, identificam um padrão consistente e estável de resposta exibido por um indivíduo, ocorrendo independentemente da situação ou do formato do instrumento (Paulhus, 1991).

Embora possam existir inúmeros estilos de resposta, alguns são mais conhecidos e estudados. Entre estes, destacam-se a Aquiescência (i.e., tendência a marcar os pontos de concordância de uma escala, independentemente de seu conteúdo), o Estilo de Respostas Extremas (i.e., utilização predominante de pontos extremos da escala, sejam eles positivos ou negativos), e o Estilo de Resposta Central (i.e., abstenção do uso de pontos extremos, utilizando apenas as opções intermediárias; [Paulhus, 1991; Ziegler, 2015]). O padrão aquiescente de resposta (ou, simplesmente, aquiescência) é um dos estilos há mais tempo estudados, e possui, na literatura científica internacional, um espaço consolidado.

Apesar disso, no Brasil o tema não é igualmente difundido. Emmuitos grupos de pesquisa brasileiros, nãoécomuma utilização de alguma medida de controle das respostas aquiescentes,
Grazielli Padilha Vieira, Daniely Fernandes Kamazaki, Cristiano Weiss, Cristian Zanon e Denise Ruschel Bandeira mesmo quando um instrumento do tipo Likert faz parte da coleta de dados. Isso pode ocorrer, entre outras coisas, pela falta de familiaridade dos pesquisadores com os métodos estatísticos ou por não estarem cientes do impacto que a aquiescência pode ter na sua pesquisa. Entende-se, dessa forma, que a abordagem aqui proposta pode contribuir para aumentar o conhecimento teórico da temática, a nível nacional, facilitando e estimulando o ensino na graduação e na pós-graduação.

Este artigo objetiva oferecer uma introdução compreensiva e abrangente ao conceito da aquiescência em instrumentos de autorrelato, atentando para as complexidades que permeiam a pesquisa sobre este estilo de resposta. Para isso, são reunidas evidências empíricas atuais e algumas discussões históricas que envolvem o fenômeno. Ao final, são discutidas algumas das soluções propostas na literatura para lidar com as respostas aquiescentes.

\section{AQUIESCÊNCIA: O CONCEITO}

0 padrão aquiescente de resposta foi um dos primeiros a ser identificado por psicometristas (Cronbach, 1942, 1946, 1950; Edwards, 1961; Messick, 1967). A aquiescência é definida como um estilo de resposta caracterizado pelo padrão sistemático de concordar com os itens de uma escala, sem considerar seu conteúdo. Na literatura, é chamada também de "yea-saying", ou "dizer sempre sim", por sua característica peculiar de respostas positivas para as afirmativas, mesmo quando são apresentados itens de conteúdos opostos (PauIhus, 1991; Valentini, 2017). Evidências provindas de diferentes delineamentos indicam que, em adultos, o padrão aquiescente tende a manter-se estável ao longo dos anos, pois se preserva em testagens longitudinais do tipo teste-reteste e com diferentes instrumentos de personalidade, atitudes, bem-estar, entre outros (Wetzel et al., 2016).

Dessa forma, autores em diferentes épocas afirmam que a presença de estilos de resposta pode ser considerada não apenas um fator prejudicial à validade dos escores, mas também um agente de compreensão para outros fenômenos psicológicos (Messick, 1967; Wetzel et al., 2016). Nessa perspectiva, se os padrões de resposta podem representar uma variável de diferença individual - possibilitando inclusive a previsão de comportamentos - não deveriam ser descartados das análises. Cronbach (1946, 1950), em suas primeiras teorizações sobre a aquiescência, mesmo enfatizando que ela tinha o potencial de levar a erros de interpretação, também sugeria a possibilidade de esta refletir um estilo individual consistente. Outros estudos iniciais sobre o fenômeno, aliás, já ponderavam que a aquiescência poderia representar a manifestação de traços de personalidade, especialmente maior extroversão e impulsividade (Couch \& Keniston, 1960; Jackson \& Messick, 1958). 


\section{H. INTERACÃO EM L PSICOLOGIA}

Historicamente, as primeiras décadas de estudos sobre a temática foram movidas por intensos debates entre os pesquisadores. Alguns autores avaliavam que a preocupação com o estilo aquiescente era injustificada (Block, 1965; Rorer, 1965), enquanto outros reforçavam a importância da identificação e do manejo dos estilos de resposta nos questionários (Messick, 1967; Ray, 1979; Rundquist, 1966). Atualmente, há um consenso entre os pesquisadores sobre o papel crítico que a aquiescência tem nas respostas às escalas (Lechner et al., 2019; Plieninger \& Heck, 2018; Zanon et al., 2018).

A aquiescência é considerada danosa por distorcer a estrutura fatorial da escala, prejudicando as evidências de validade convergente e discriminante do construto investigado (i.e., por produzir escores inapropriados, Lechner \& Rammste$\mathrm{dt}, 2015)$. Mais especificamente, a aquiescência pode produzir um fator espúrio adicional na estrutura interna de instrumentos de medida não relacionado ao construto de interesse (ver Soto et al., 2008, para uma demonstração visual do impacto do fator de aquiescência na estrutura do Big Five Inventory). Ou seja, a aquiescência pode influenciar, substancialmente, os resultados de análises de estrutura interna, consistência interna, a adequação dos itens às respectivas facetas teóricas, assim como os parâmetros encontrados nas amostras (Danner et al., 2015; Zanon et al., 2018). O problema torna-se ainda mais indesejável para pesquisadores com o objetivo de comparar grupos, pois estes podem apresentar níveis diferentes de aquiescência (Lechner et al., 2019).

Até o momento, diversos fatores já foram associados à aquiescência. Cronbach $(1942,1946)$ inaugurou o debate sugerindo que a tendência aquiescente é mais suscetível a aparecer frente a itens difíceis ou ambíguos, quando o indivíduo não sabe o que responder ou fica em dúvida quanto à resposta correta. Também foram levantadas hipóteses acerca das motivações sociais possivelmente subjacentes a esse estilo (Diers, 1964; Knowles \& Nathan, 1997).

Hoje se admite que a aquiescência tende a ocorrer de maneira involuntária, sem a real intencionalidade do participante (Knowles \& Condon, 1999; Lechner et al., 2019). Não se pode afirmar, no entanto, que esteja relacionado a uma forma descuidada ou desatenta de responder (Baumgartner et al., 2018; Kam \& Meyer, 2015). Segundo Grau et al. (2019), mesmo que seja possível observar sobreposições entre o estilo aquiescente e a forma descuidada de responder (careless responding), estes são construtos distintos que devem operar por diferentes mecanismos. A seguir, apresentam-se algumas das discussões referentes às abordagens motivacionais e cognitivas da aquiescência.
Grazielli Padilha Vieira, Daniely Fernandes Kamazaki, Cristiano Weiss, Cristian Zanon e Denise Ruschel Bandeira

\section{PERSPECTIVAS CLÁSSICAS: ABORDAGENS MOTIVACIONAL E COGNITIVA Ả AQUIESCÊNCIA}

\section{ABORDAGEM MOTIVACIONAL}

A tendência de avaliar a si mesmo influenciado por um viés motivacional (i.e., por necessidade de adequação social; reflexo de normais sociais) é um problema identificado há bastante tempo na pesquisa sobre padrões de resposta. Um estilo de resposta que, assim como a aquiescência, tem uma história longa de estudos é a desejabilidade social (Edwards, 1957; Paulhus, 1984). Definida inicialmente por Edwards (1957), a resposta socialmente desejável é considerada aquela em que o indivíduo endossa itens com conteúdo socialmente desejável (e.g., ser feliz) e discorda de itens com conteúdo indesejável (e.g., características antissociais).

A possível relação ou sobreposição da aquiescência com a desejabilidade social foi uma das questões que mais motivaram as pesquisas iniciais sobre esses estilos de resposta (Diers, 1964; Edwards, 1961). Para alguns autores, apesar de reconhecidos como dois fenômenos distintos, eles poderiam coexistir ou até mesmo agir por mecanismos similares. Além disso, ambos pareciam sofrer influência da suposta aprovação ou desaprovação social, considerando que os itens costumam descrever opiniões e comportamentos associados a normas sociais (Diers, 1964).

Edwards (1961) argumentou, apresentando um estudo de caso, que a tendência à desejabilidade social podia ser tão dominante que acabaria por impedir e mascarar o resultado aquiescente. Assim, o que muitos pesquisadores consideravam ser apenas influência da aquiescência seria um resultado produzido pelo efeito da desejabilidade social (Edwards, 1961). Couch e Keniston (1961) apresentaram evidências de que a tendência aquiescente poderia ser considerada independente de qualquer aspecto relativo à desejabilidade social. Outros estudos também apontaram uma falta de correlação entre os dois estilos (Diers, 1964; Greenwald \& Clausen, 1970).

Ferrando e Anguiano-Carrasco (2010) tentaram esclarecer a antiga controvérsia sobre o impacto conjunto dos dois estilos nas respostas aos itens. Em um estudo empírico com 2.322 universitários (média de idade 22 anos, $75 \%$ mulheres), os autores contrariaram a ideia de Edwards (1961) sobre a predominância da desejabilidade social em detrimento da aquiescência. Os achados indicaram que a resposta aquiescente opera em todos os tipos de itens, inclusive naqueles imbuídos de desejabilidade social.

Em relação aos mecanismos envolvidos, evidências recentes indicam que a desejabilidade social parece se desenvolver de forma um pouco diferente do que os outros estilos de resposta, pois é o único que demanda uma leitura minuciosa de 


\section{MLE INTERACÃO EM PSICOLOGIA}

todos os itens (Grau et al., 2019). Outros estudos que investigaram aspectos motivacionais e cognitivos na aquiescência não mostraram esse estilo relacionado a preocupações sociais de qualquer ordem (Knowles \& Condon, 1999; Knowles $\&$ Nathan, 1997). Em vez disso, esses autores sugeriram que a aquiescência surge de falhas no processamento cognitivo envolvido na ação de responder os itens em um questionário.

\section{ABORDAGEM COGNITIVA}

As primeiras conceitualizações do padrão aquiescente, feitas por Cronbach (1942, 1946), sugeriam que o problema deveria estar ligado a um mecanismo do processo cognitivo. Segundo o autor, a ação de concordar com um item seria feita de maneira acrítica, especialmente quando o respondente julga o item como ambíguo ou difícil de responder. Por essa visão, as motivações sociais não estariam realmente no cerne do fenômeno aquiescente. Adotando uma abordagem cognitiva, no entanto, diferentes modelos já foram elaborados e múltiplos mecanismos considerados relevantes para a compreensão desse estilo de resposta.

Alguns autores relataram um possível viés de confirmação produzido pela busca de informações na memória (Zuckerman et al., 1995). Ou seja, indivíduos mais aquiescentes tendem a concordar com uma afirmação se forem capazes de lembrar mais facilmente de um ou dois fatos que a confirmem. Em vez de refletir sobre todas as informações disponíveis na memória, eles teriam a tendência de usar rotas heurísticas que acabariam enviesando suas respostas (Schwarz et al., 1991). De forma semelhante, Knowles e Condon (1999) teorizaram que a aquiescência seria resultado de uma falha no processo de reconsideração do item, pois este requer maior esforço mental. Uma sobrecarga na capacidade cognitiva do respondente, por qualquer motivo que seja (e.g., distratores, estresse), impediria a devida reconsideração da afirmação, levando à aceitação automática da mesma.

Outros estudos propuseram teorizações mais específicas e detalhadas sobre o processo cognitivo envolvido na ação de responder questionários com escalas Likert. Tourangeau e Rasinski (1988) apresentaram um modelo de quatro estágios, que podem ser resumidos em "interpretação", "recuperação", "julgamento" e "resposta". Shulruf et al. (2008) propuseram um modelo compreensivo de cinco estágios, contemplando a possibilidade de "edição" da resposta por influência da desejabilidade social e o acesso a informações de ambiente e contexto.

Dentro dessa abordagem, entende-se que podem ocorrer falhas durante os estágios, pois o processo requer certa sofisticação cognitiva. Dessas falhas surgiriam os estilos de resposta (Shulruf et al., 2008; Zanon et al., 2018). No entanto, assim como os autores divergem em relação à essência e quantidade desses estágios cognitivos, não há consenso
Grazielli Padilha Vieira, Daniely Fernandes Kamazaki, Cristiano Weiss, Cristian Zanon e Denise Ruschel Bandeira também quanto ao ponto específico que dá origem aos estilos de resposta (Böckenholt, 2019; Plieninger \& Heck, 2018).

Nesse sentido, a construção dos itens de um questionário (e.g., afirmativos, negativos, opostos) também podem ser considerados importantes para entender a aquiescência (Baumgartner et al., 2018; Böckenholt, 2019). Há autores que argumentam que o estilo aquiescente se manifesta na presença de todos os tipos de itens (Hutton, 2017; Weijters \& Baumgartner, 2012). No entanto, segundo Böckenholt (2019), escalas que contêm de forma conjunta itens afirmativos e opostos tendem a apresentar menores índices de resposta aquiescente. Para Baumgartner et al. (2018), a aquiescência pode ser um simples erro de resposta causado pela má compreensão dos itens que compõem a escala. Segundo estes autores, itens opostos e na forma de negação, por exemplo, são mais difíceis de interpretar e responder, gerando maiores índices de aquiescência.

\section{PROPOSTAS INTEGRATIVAS PARA COMPREENSÃO DA AQUIESCÊNCIA E SEUS CORRELATOS}

A pesquisa em torno do estilo aquiescente tem investigado diferentes correlatos em três grandes áreas: (a) cultural (e.g., normas sociais, desenvolvimento econômico), (b) individual (e.g., idade, escolaridade, valores pessoais) e (c) situacional (e.g., privacidade, interesse na tarefa). Ao longo do tempo, os autores têm utilizado diferentes teorias para fundamentar os correlatos associados à aquiescência. He e Van de Vijver (2016) discutem seus achados, por exemplo, ressaltando o papel do nível educacional na sofisticação cognitiva, condição que influencia o processo mental envolvido ao responder uma escala Likert. No entanto, também admitem a influência de uma estratégia de gerenciamento de impressões (impression management), que indica o objetivo do respondente em causar uma certa impressão no entrevistador e/ou demais pessoas presentes (He \& Van de Vijver, 2016).

Liu et al. (2017), ao compararem levantamentos online e face a face, incluíram a análise da variável de raça e etnia, além de promover uma discussão pelo viés do gerenciamento de impressões. Nesse caso, entende-se que tradições culturais relacionadas a normas sociais, as quais estão alinhadas a características individuais (valores), podem permitir interpretações de abordagem motivacional. Por outro lado, a variação da aquiescência pode ser interpretada pelo viés cognitivo quando se refere a questões como idade ou escolaridade (He \& Van de Vijver, 2016; Lechner et al., 2019).

Recentemente, Lechner et al. (2019) propuseram um modelo integrativo para a compreensão da resposta aquiescente, indicando uma ação compartilhada pelas múltiplas variáveis de correlatos. No modelo, os autores postulam dois mecanis- 


\section{MLE INTERACÃO EM PSICOLOGIA}

mos através dos quais supostamente esses aspectos se relacionam com a aquiescência: "capacidade de processamento cognitivo" e "estilos de comunicação de deferência" (este último relativo a padrões de interação social, com ênfase no respeito a figuras de autoridade). Assim, os diferentes correlatos podem ser explicados, na maioria das vezes, por um destes mecanismos - ou ambos (Lechner et al., 2019).

A seguir serão apresentadas, separadamente, evidências dos principais correlatos investigados na pesquisa sobre aquiescência. Serão destacadas também, brevemente, algumas das proposições teóricas dos autores na discussão dos achados.

\section{CORRELATOS CULTURAIS}

Há robusta literatura indicando forte relação de valores culturais e normas sociais com o padrão aquiescente, além de outras variáveis de nível nacional, como desenvolvimento econômico, liberdade política, corrupção e desempenho educacional médio (Lechner et al., 2019; Rammstedt et al., 2017). 0 trabalho de Lechner et al. (2019), com dados de 60 países heterogêneos (retirados da World Values Survey, N = 90.347), ilustra o quão grande pode ser a variação nos índices de aquiescência entre culturas. Os autores indicam que em nações como Holanda, Suécia e Alemanha a aquiescência é menos presente, enquanto Egito, Qatar, Líbia e Namíbia apresentam maiores níveis. Outros autores também já apontaram para níveis mais elevados na Europa meridional (e.g., Portugal, Grécia) e menores no Norte europeu (e.g., Dinamarca, Noruega; He \& Van de Vijver, 2016; Rammstedt et al., 2017).

Resultados de Rammstedt et al. (2017) indicaram uma variância positivamente significativa da aquiescência relacionada aos maiores níveis de corrupção e a formas de estruturação mais coletivista dos países. Para Lechner et al. (2019), no entanto, o padrão aquiescente é mais bem explicado por fortes normas sociais de deferência (i.e., atitude de respeito à autoridade), do que em função da situação nacional de corrupção ou de desenvolvimento econômico. Nesse sentido, um estudo realizado em Taiwan indicou que o estilo de não confrontação na resolução de conflitos demonstra uma relação positiva com a aquiescência (Chen et al., 2017).

Entre as grandes áreas temáticas de correlatos da aquiescência, entretanto, as questões culturais não são consideradas as mais significativas para alguns autores (He \& Van de Vijver, 2016; Rammstedt et al., 2017). No estudo de He e Van de Vijver (2016), por exemplo, os estilos de resposta estavam mais associados a um fator individual: sofisticação cognitiva. Já no trabalho de Rammstedt et al. (2017), embora as diferenças culturais tenham explicado $15 \%$ da variância da aquiescência, os outros $85 \%$ foram motivados por variáveis individuais.
Grazielli Padilha Vieira, Daniely Fernandes Kamazaki, Cristiano Weiss, Cristian Zanon e Denise Ruschel Bandeira

\section{CORRELATOS INDIVIDUAIS}

As principais características individuais apontadas como correlatos significativos da aquiescência são: idade, nível educacional, valores pessoais e sexo (He \& Van de Vijver, 2016; Lechner et al., 2019; Rammstedt et al., 2017). Em relação à idade, a investigação conduzida por Lechner et al. (2019), com dados de mais de 90 mil participantes, indicou uma associação positiva não linear com o estilo aquiescente. Nesse estudo, o padrão de resposta foi maior entre respondentes mais velhos (acima de 55 anos), seguido por jovens e adolescentes (16-24 anos). A faixa etária menos afetada foi 45-54 anos (Lechner et al., 2019). $\mathrm{Na}$ esfera educacional, a aquiescência é menos prevalente entre indivíduos com educação secundária e pós-graduação em comparação com aqueles que possuem apenas formação primária (Lechner et al., 2019; Rammstedt et al., 2017).

Alguns autores argumentam que indivíduos com baixo nível educacional e médias inferiores de habilidade cognitiva são menos familiarizados com material verbal e pensamento abstrato, o que pode produzir respostas mais aquiescentes (He \& Van de Vijver, 2016; Lechner et al., 2019). Nesse sentido, uma idade mais avançada e menor educação formal são fatores que prejudicam a capacidade de processamento cognitivo envolvido na ação de responder um questionário, tornando esses grupos mais passíveis de desenvolver estilos de resposta (Knowles \& Condon, 1999; Lechner et al., 2019; Lechner \& Rammstedt, 2015). Para He e Van de Vijver (2016), os indivíduos com maior sofisticação cognitiva costumam moderar suas respostas, expressando-se com mais nuances.

Alguns estudos indicam uma tendência levemente maior de estilo aquiescente em indivíduos do sexo masculino (He et al., 2014; Lechner et al., 2019; Rammstedt et al., 2017), mas a origem dessa diferença ainda não é clara. Já os valores pessoais, como deferência (Lechner et al., 2019) ou baixo grau de conservadorismo (Rammstedt et al., 2017), vistos como uma característica de diferença individual, relacionam-se positivamente com a aquiescência. Para Lechner et al. (2019), os valores individuais podem ter um papel importante durante a ação de responder um instrumento, influenciando a experiência do respondente.

\section{CORRELATOS SITUACIONAIS}

A coleta dos dados de uma pesquisa está sempre cercada de situações que podem ou não influenciar o resultado obtido. Um antigo estudo de Lenski e Leggett (1960) associou a maior diferença de status quo entre pesquisador e respondente com maiores índices de aquiescência. Os autores, à época, já recomendavam cuidado com a relação social invariavelmente criada durante uma pesquisa. Em um achado semeIhante, verificou-se níveis mais elevados de aquiescência em 


\section{MLE INTERACÃO EM PSICOLOGIA}

relatos coletados por entrevistadores experientes do que por entrevistadores inexperientes (Olson \& Bilgen, 2011). Nesse sentido, entende-se que a dinâmica interativa estabelecida entre o participante e o pesquisador pode ter uma parcela significativa de responsabilidade no surgimento dos vieses de resposta (Liu et al., 2017).

O nível de engajamento do respondente com a tarefa também tem sido considerado um fator que pode influenciar o surgimento de respostas aquiescentes. Lechner et al. (2019) indicaram que, inesperadamente, os indivíduos que apresentaram maior aquiescência são os que demonstraram maior interesse na entrevista. Os autores sugerem que o interesse aumentado pode refletir em uma interdependência forte entre entrevistador e entrevistado, estimulando valores relacionados à deferência (no nível individual).

Outros achados indicam diferenças importantes dos questionários preenchidos pela internet ou por meio de computador quando comparados ao modelo tradicional de papel e caneta (Weijters et al., 2008). Embora ambos os métodos sejam suscetíveis aos estilos de resposta, uma investigação avaliando os dois formatos indicou que as coletas face a face se mostram mais vulneráveis ao estilo aquiescente, independente da raça ou etnia do respondente (Liu et al., 2017). A explicação pode estar relacionada a achados que indicam a presença de outras pessoas como fator passível de induzir respostas mais aquiescentes (He \& Van de Vijver, 2016) ou socialmente desejáveis (Richman et al., 1999).

Além da presença do entrevistador e, eventualmente, de terceiros, a coleta presencial também está mais sujeita a outros distratores, como barulhos externos. Dessa forma, pode-se interpretar que a experiência da testagem presencial aumenta a demanda cognitiva de modo geral, elevando as chances de respostas aquiescentes (He \& Van de Vijver, 2016; Knowles \& Condon, 1999). Lechner et al. (2019) obtiveram achados que corroboram essa ideia, pois observaram uma diminuição da aquiescência relacionada ao aumento da privacidade na aplicação do instrumento.

Entretanto, a influência dos múltiplos fatores situacionais nos estilos de resposta, embora positiva, tem-se mostrado pouco relevante em comparação com os demais correlatos $(\mathrm{He}$ $\&$ Van de Vijver, 2016; Lechner et al., 2019). Esses autores sugerem que essas conjunturas específicas desempenham um papel pequeno no desenvolvimento de respostas aquiescentes e, além disso, são fatores que podem ser facilmente controlados.

\section{MÉTODOS DE CONTROLE DE RESPOSTA AQUIESCENTE}

Pesquisas na área da personalidade, especialmente, têm produzido alguns métodos para lidar com os estilos de respostas. Alguns procedimentos foram propostos para controlar os
Grazielli Padilha Vieira, Daniely Fernandes Kamazaki, Cristiano Weiss, Cristian Zanon e Denise Ruschel Bandeira efeitos da aquiescência, em especial, a ipsatização (Soto et al., 2008) e o modelo do fator comum com intercepto randômico (Maydeu-Olivares \& Coffman, 2006), entre outros métodos.

A ipsatização provê uma solução de escores individuais para cada sujeito. Assim, é um tipo de padronização intrassujeitos que faz uso de pares de itens positivos e negativos (i.e., reversos) para avaliar cada fator. A padronização das diferenças da aquiescência é feita para cada indivíduo, através da classificação baseada na média e desvio-padrão dos escores brutos dos grupos de pares de itens. Ou seja, os dados brutos são subtraídos de uma média dos pares de itens (i.e., positivos e negativos) e divididos pelo desvio-padrão deste mesmo conjunto de itens (Soto et al., 2008). O conjunto resultante de respostas recodificadas produz uma nova variável com média de 0 e desvio padrão de 1 (Soto et al., 2008; Zanon et al., 2018). Quanto mais alto o escore, mais elevado é o valor do traço latente de cada participante e vice-versa.

O segundo método, mais complexo, é o modelo do fator comum com intercepto randômico (Maydeu-Olivares \& Coffman, 2006). Para compreender este modelo, faz-se necessário entender, primeiramente, o que é o fator comum e o intercepto randômico. 0 fator comum pressupõe que existe uma variável latente ou fator subjacente (hipotético), que explica um certo conjunto de variáveis observadas. Esse fator latente é a variável psicológica que interessa avaliar (e.g., habilidade aritmética, leitura e vocabulário teriam a inteligência como fator comum). Ou seja, um grupo de variáveis correlacionadas entre si são explicadas por um fator comum (Brown, 2015). Assim, a carga fatorial estimada representa um coeficiente de regressão que, se padronizado, indica a força e o sentido da relação entre fator e variável observada (Zanon et al., 2020). Ao se utilizar uma escala de personalidade (e.g., Big Five), os itens seriam explicados pelos cinco fatores correspondentes aos fatores de personalidade. Entretanto, a aquiescência pode produzir um fator adicional espúrio a esta solução, enviesar as cargas fatoriais dos itens e, assim, fazer com que a escala apresente parâmetros inapropriados, principalmente se a escala foi construída em polos positivos (Danner et al., 2015).

No modelo do fator comum, assume-se que o conjunto de variáveis observadas representam funções lineares da relação entre o fator comum com os itens e erros aleatórios. Pressupõe-se também que a relação entre cada variável observada e os fatores comuns são constantes para todos os respondentes. Ou seja, os interceptos e cargas fatoriais do modelo não variam entre os respondentes (Maydeu-Olivares \& Coffman, 2006). 0 intercepto (i.e., média estimada do item) é o ponto onde a linha (da função linear) intersecta o eixo y, sendo sempre constante (Kline, 2015). Entretanto, as respostas dos participantes podem variar muito dentro de uma escala de tipo Likert, e seus estilos de respostas podem influenciar os escores de formas diversas, tornando a suposição de co- 


\section{M." INTERACÃO EM PSICOLOGIA}

eficientes invariantes nem sempre confiável (Maydeu-Olivares \& Coffman, 2006). Por isso, esses autores propuseram um modelo em que o intercepto do modelo pode variar entre os indivíduos, mas não entre os itens. Ou seja, os interceptos são tratados como um efeito aleatório que pode variar entre os participantes. Nesse modelo, contudo, estima-se uma variância geral, sendo que os interceptos individuais não são computados para cada participante.

Em suma, o modelo do fator comum com o intercepto randômico modela diferenças individuais sistemáticas não previstas pelo tradicional modelo do fator comum - usado na maior parte dos estudos de estrutura interna de escalas psicológicas. Esse procedimento permite ao intercepto variar de respondente a respondente mesmo que seja o mesmo para todos os itens. 0 escore encontrado não visa a explicar as diferenças individuais, mas estima uma variável aleatória que compreende um fator que não se correlaciona aos fatores descritos pelos itens que se deseja medir, podendo explicar a aquiescência (Maydeu-Olivares \& Coffman, 2006).

No Brasil, um estudo sugeriu a superioridade do método com interceptos randômicos em relação ao método de ipsatização para o controle da aquiescência (Zanon et al., 2018). Uma explicação para esse resultado é que o modelo com o intercepto randômico foi desenvolvido para controlar diversos estilos de resposta. A proposta de Soto et al. (2008), por sua vez, foi elaborada apenas para o controle da aquiescência. Assim, o procedimento de Maydeu-Olivares e Coffman (2006) parece ser mais promissor para estimação de modelos baseados no Big Five.

Billiet e McClendon (2000) mostraram que é possível controlar a aquiescência utilizando modelagem de equações estruturais, especificando um modelo com um fator geral de aquiescência. Nessa proposta os autores sugerem que sejam discriminados dois fatores de conteúdo dos itens ( $\eta 1$ e $\eta 2$ ) mais um fator de estilo (n3). São carregadas cargas fixas de +1 nos fatores para que as respostas a todos os itens sejam afetadas da mesma forma pelo estilo de resposta (aquiescência). Assim, não é esperado uma relação entre conteúdo e o estilo de resposta, por isso, as covariâncias entre os dois fatores de conteúdo e o fator de estilo são definidos como zero. Os autores utilizaram dois conjuntos de 14 itens balanceados, que eram contraditórios em seus significados, mas não necessariamente opostos ou contrários.

Após isso, a variável latente foi correlacionada com uma variável medida pelo número de concordâncias no conjunto de itens balanceados (pontuação para aquiescência). A forte correlação $(0,90)$ entre as duas variáveis indica que são duas medidas do mesmo conceito, aquiescência. Essa proposta parece mais parcimoniosa do que a proposta do intercepto randômico (Maydeu-Olivares \& Coffman, 2006), pois estima
Grazielli Padilha Vieira, Daniely Fernandes Kamazaki, Cristiano Weiss, Cristian Zanon e Denise Ruschel Bandeira uma variável relacionada à pontuação da aquiescência, e não uma variável aleatória que pode ser a aquiescência (Billiet \& McClendon, 2000).

Por último, outra proposta utilizou simulação computacional avaliando os efeitos do aumento de respostas aleatórias e fixas na avaliação de duas escalas de controle de estilo de respostas com suas contrapartes do questionário Minnesota Multiphasic Personality Inventory-2 Restructured Form-MMPI-2-RF (Handel et al, 2010). Os autores também revisaram como os escores para a escala Restructured Clinical (RC) são afetados por respostas não baseada em conteúdo, sendo que os resultados mostraram que a $\mathrm{RC}$ apresenta coeficientes robustos diante desses tipos de resposta, como a aquiescência e contra-aquiescência. Esse método de simulação permite a identificação da validade dos instrumentos para identificar o estilo de resposta aquiescente (Handel et al., 2010).

\section{CONSIDERAÇÕES FINAIS}

Este manuscrito reforça a importância de discutir teoricamente o fenômeno dos estilos de resposta e de identificar esses padrões em estudos futuros. As complexidades do estilo aquiescente, apresentadas neste trabalho, devem ser consideradas durante o desenvolvimento de novos instrumentos que visem atingir um padrão-ouro internacional, assim como nos processos de adaptação transcultural. Entende-se, ainda, que o controle dos estilos de resposta deve ser incorporado pelos grupos de pesquisa quando os estudos fazem uso de instrumentos com escalas Likert. Em concordância com Zanon et al. (2018), sugere-se que o controle da aquiescência seja realizado a partir da modelagem de interceptos randômicos, uma vez que este tem se mostrado mais robusto que o método de ipsatização. Além disso, o modelo de Maydeu-Olivares e Coffman (2006) é capaz de controlar também outros padrões de respostas, ampliando a segurança dos pesquisadores na obtenção de resultados confiáveis de um instrumento.

Não se pode deixar de recordar, entretanto, as potencialidades da aquiescência como um construto passível de informar diferenças individuais (e.g., personalidade, cultura, habilidades cognitivas). Um caminho possível para futuras pesquisas, nesse sentido, é investigar a relação entre o estilo aquiescente e as psicopatologias. Alguns pesquisadores, por exemplo, já têm aplicado esse conceito visando compreender melhor as características de pacientes. Dentro dessa perspectiva, encontram-se estudos como o de Finlay e Lyons (2002), que discute a tendência aquiescente em pessoas com deficiência intelectual, ou o de Ross e Mirowsky (1984), que apontou para a relação entre estresse psicológico e respostas socialmente desejáveis (mas não aquiescentes). Em outro estudo com proposta semelhante, Johnson et al. (1975) demonstraram a associação positiva entre a gravidade da esquizofrenia e os níveis de aquiescência. 


\section{WINTERACCÃO EM PSICOLOGIA}

Assim, cabe destacar que o estudo do fenômeno aquiescente vai muito além do seu papel no desenvolvimento e na utilização de instrumentos de avaliação. 0 estudo desse e de outros estilos de resposta também permite a investigação de correlatos psicopatológicos e teorizações sobre o impacto de mecanismos psicológicos típicos e atípicos no desenvolvimento e na avaliação psicológica. Logo, a aplicação dos conhecimentos acerca da aquiescência e de seus métodos de controle pode possibilitar a construção de uma pesquisa científıca mais qualificada no cenário brasileiro.

\section{DECLARAÇÃO DA CONTRIBUIÇÃO DOS AUTORES}

Certificamos que todos os autores participaram suficientemente do trabalho para tornar pública sua responsabilidade pelo conteúdo. A contribuição de cada autor pode ser atribuída como se segue:

G.P.V e D.F.K foram responsáveis pela idealização do artigo, administração do projeto, conceitualização, investigação, construção da metodologia, preparação do rascunho original. C.W. Contribuiu para investigação, construção da metodologia, preparação do rascunho original. C.Z. contribuiu para revisão, edição do manuscrito e supervisão. G.P.V., D.F.K. e C.W. realizaram a escrita, edição e revisão da versão final do manuscrito. D.R.B foi responsável pela supervisão do artigo, revisão e aprovação da versão final do manuscrito.

\section{DECLARAÇÃO DE CONFLITOS DE INTERESSE}

Os autores declaram que não há conflitos de interesse no manuscrito submetido.

\section{DECLARAÇÃO DE FINANCIAMENTO}

A pesquisa relatada no manuscrito foi financiada parcialmente pela bolsa de mestrado da primeira autora (CNPq), da segunda autora (CAPES) e do terceiro autor (CNPq).

\section{REFERÊNCIAS}

Baumgartner, H., Weijters, B., \& Pieters, R. (2018). Misresponse to Survey Questions: A conceptual framework and empirical test of the effects of reversals, negations, and polar opposite core concepts. Journal of Marketing Research, 55(6), 869-883. https://doi.org/10.1177/0022243718811848

Billiet, J. B., \& McClendon, M. J. (2000). Modeling acquiescence in measurement models for two balanced sets of items. Structural equation modeling, 7(4), 608-628. https://doi. org/10.1207/s15328007sem0704_5
Grazielli Padilha Vieira, Daniely Fernandes Kamazaki, Cristiano Weiss, Cristian Zanon e Denise Ruschel Bandeira
Block, J. (1965). The challenge of response sets: Unconfounding meaning, acquiescence, and social desirability in the MMPI. Appleton-Century-Crofts.

Böckenholt, U. (2019). Contextual responses to affirmative and/or reversed-worded items. Psychometrika, 84(4), 986999. https://doi.org/10.1007/s11336-019-09680-7

Brown, T. A. (2015). Confirmatory factor analysis for applied research (2nd ed.). The Guilford Press.

Chen, H. C., Chen, I. H., Lin, S. Y., \& Chen, Y. (2017). Cultural Influences in Acquiescent Response: A study of trainer evaluation biases. International Journal of Selection and Assessment, 25(1), 1-10. https://doi.org/10.1111/ijsa.12155

Couch, A., \& Keniston, K. (1960). Yeasayers and naysayers: Agreeing response set as a personality variable. Journal of Abnormal and Social Psychology, 60(2), 151-174. https:// doi.org/10.1037/h0040372

Couch, A., \& Keniston, K. (1961). Agreeing response set and social desirability. Journal of Abnormal and Social Psychology, 62(1), 175-179. https://doi.org/10.1037/h0047429

Cronbach, L. J. (1942). Studies of acquiescence as a factor in the true-false test. Journal of Educational Psychology, 33(6), 401-415. https://doi.org/10.1037/h0054677

Cronbach, L. J. (1946). Response sets and test validity. Educational and psychological measurement, 6(4), 475-494. https://doi.org/10.1177/001316444600600405

Cronbach,L.J.(1950). Furtherevidenceonresponsesetsandtest design. Educational and Psychological Measurement, 10(1), 3-31. https://doi.org/10.1177/001316445001000101

Danner, D., Aichholzer, J., \& Rammstedt, B. (2015). Acquiescence in personality questionnaires: Relevance, domain specificity, and stability. Journal of Research in Personality, 57, 119-130. https://doi.org/10.1016/j.jrp.2015.05.004

Diers, C. J. (1964). Social desirability and acquiescence in response to personality items. Journal of Consulting Psychology, 28(1), 71-77. https://doi.org/10.1037/h0043753

Edmondson, D. R. (2005, April 28-May1). Likert scales: A history. Proceedings of the 12th Conference on Historical Analysis and Research in Marketing (CHARM), University of South Florida.

Edwards, A. L. (1957). The social desirability variable in personality assessment and research. Dryden Press.

Edwards, A. L. (1961). Social desirability or acquiescence in the MMPI? A case study with the SD Scale. The Journal of Abnormal and Social Psychology, 63(2), 351-359. https:// doi.org/10.1037/h0045005

Ferrando, P. J., \& Anguiano-Carrasco, C. (2010). Acquiescence and social desirability as item response determinants: An IRT-based study with the Marlowe-Crowne and the EPQ Lie scales. Personality and Individual Differences, 48(5), 596-600. https://doi.org/10.1016/j.paid.2009.12.013 
Finlay, W. M., \& Lyons, E. (2002). Acquiescence in interviews with people who have mental retardation. Mental retardation, 40(1), 14-29. https://doi.org/10.1352/ 0047-6765(2002)040<0014:AlIWPW>2.0.C0;2

Grau, I., Ebbeler, C., \& Banse, R. (2019). Cultural differences in careless responding. Journal of Cross-Cultural Psychology, 50(3), 336-357. https://doi. org/10.1177/0022022119827379

Greenwald, H. J., \& Clausen, J. D. (1970). Test of relationship between yeasaying and social desirability. Psychological Reports, 27(1), 139-141.https://doi.org/10.2466/ pr0.1970.27.1.139

Handel, R. W., Ben-Porath, Y. S., Tellegen, A., \& Archer, R. P. (2010). Psychometric functioning of the MMPI-2-RF VRIN-r and TRIN-r scales with varying degrees of randomness, acquiescence, and counter-acquiescence. Psychological assessment, 22(1), 87-95. https://doi.org/10.1037/ a0017061

He, J., \& Van de Vijver, F. J. R. (2016). Response styles in factual items: Personal, contextual and cultural correlates. International Journal of Psychology, 51, 445-452. https://doi. org/10.1002/ijop.12263

He, J., Bartram, D., Inceoglu, I., \& Van de Vijver, F. J. R. (2014). Response styles and personality traits. Journal of Cross-Cultural Psychology, 45(7), 1028-1045. https://doi. org/10.1177/0022022114534773

Hutton, A. C. (2017). Assessing acquiescence in surveys using positively and negatively worded questions. [Dissertação de mestrado não publicada, Master of Fine Arts, Virginia Commonwealth University]. https://scholarscompass. vcu.edu/etd/4679

Jackson, D. N., \& Messick, S. (1958). Content and style in personality assessment. Psychological Bulletin, 55, 243-252. https://doi.org/10.1037/h0045996

Johnson, J. E., Petzel, T. P., Becker, P. A., \& Rudinsky, M. F. (1975). Degree of pathology as related to readiness to accept labels in process and reactive schizophrenics. International Journal of Social Psychiatry, 21(4), 292-296. https://doi.org/10.1177/002076407502100408

Joshi, A., Kale, S., Chandel, S., \& Pal, D. K. (2015). Likert scale: Explored and explained. British Journal of Applied Science \& Technology, 7(4), 396-403. https://doi.org/10.9734/ BJAST/2015/14975

Kam, C. C. S., \& Meyer, J. P. (2015). How careless responding and acquiescence response bias can influence construct dimensionality. Organizational Research Methods, 18(3), 512-541. https://doi.org/10.1177/1094428115571894

Kline, R. B. (2015). Principles and practice of structural equation modeling. The Guilford Press.

Knowles, E. S., \& Condon, C. A. (1999). Why People say "yes": A dual-process theory of acquiescence. Journal of Personality and Social Psychology, 77(2), 379. https://doi. org/10.1037/0022-3514.77.2.379
Knowles, E. S., \& Nathan, K. T. (1997). Acquiescent Responding in Self-reports: Cognitive style or social concern?. Journal of Research in Personality, 31(2), 293-301. https://doi. org/10.1006/jrpe.1997.2180

Lechner, C. M., \& Rammstedt, B. (2015). Cognitive ability, acquiescence, and the structure of personality in a sample of older adults. Psychological Assessment, 27, 1301-1311. https://doi.org/10.1037/pas0000151

Lechner, C. M., Partsch, M. V., Danner, D., \& Rammstedt, B. (2019). Individual, situational, and cultural correlates of acquiescent responding: Towards a unified conceptual framework. British Journal of Mathematical and Statistical Psychology, 72(3), 1-21. https://doi.org/10.1111/ bmsp. 12164

Lenski, G. E., \& Leggett, J. C. (1960). Caste, class, and deference in the research interview. American Journal of Sociology, 65, 463-467. https://doi.org/10.1086/222750

Liu, M., Conrad, F. G., \& Lee, S. (2017). Comparing acquiescent and extreme response styles in face-to-face and web surveys. Quality \& Quantity, 51(2), 941-958. https://doi. org/10.1007/s11135-016-0320-7

Maydeu-Olivares, A., \& Coffman, D. L. (2006). Random intercept item factor analysis. Psychological Methods, 11(4), 344-362. https://doi.org/10.1037/1082-989X.11.4.344

Messick, S. (1967). The psychology of acquiescence: An interpretation of research evidence. In: I. A. Berg (Ed.), Response set in personality assessment (pp. 115-145). Aldine. https://doi.org/10.1002/j.2333-8504.1966.tb00357.x

Olson, K., \& Bilgen, I. (2011). The role of interviewer experience on acquiescence. Public Opinion Quarterly, 75(1), 99-114. https://doi.org/10.1093/poq/nfq067

Paulhus, D. L. (1984). Two-component models of socially desirable responding. Journal of Personality and Social Psychology, 46(3), 598-609. https://doi.org/10.1037/00223514.46.3.598

Paulhus, D. L. (1991). Measurements and control of response bias. In: J. P. Robinson, P. R. Shaver, \& L. S. Wrightsman (Eds.), Measures of personality and social psychological attitudes (pp. 17-59). Academic Press. https://doi. org/10.1016/B978-0-12-590241-0.50006-X

Plieninger, H., \& Heck, D. W. (2018). A new model for acquiescence at the interface of psychometrics and cognitive psychology. Multivariate behavioral research, 53(5), 633-654. https://doi.org/10.1080/00273171.2018.1469966

Rammstedt, B., Danner, D., \& Bosnjak, M. (2017). Acquiescence Response Styles: A multilevel model explaining individual-level and country-level differences. Personality and Individual Differences, 107, 190-194. https://doi.org/10.1016/j. paid.2016.11.038

Ray, J. J. (1979). Is the acquiescent response style problem not so mythical after all? Some results from a successful balanced F scale. Journal of Personality Assessment, 43(6), 638-643. https://doi.org/10.1207/s15327752jpa4306_14 


\section{-4: INTERACÃO EM LFICOLOGIA}

Richman, W. L., Kiesler, S., Weisband, S., \& Drasgow, F. (1999). A meta-analytic study of social desirability distortion in computer-administered questionnaires, traditional questionnaires, and interviews. Journal of Applied Psychology, 84(5), 754-775. https://doi.org/10.1037/0021-9010.84.5.754

Rorer, L. G. (1965). The great response-style myth. Psychological Bulletin, 63(3), 129-156. https://doi.org/10.1037/ h0021888

Ross, C. E., \& Mirowsky, J. (1984). Socially-desirable response and acquiescence in a crosscultural survey of mental health. Journal of Health and Social Behavior, 25(2), 189-197. https://doi.org/10.2307/2136668

Rundquist, E. A. (1966). Item and response characteristics in attitude and personality measurement: $A$ reaction to $L$. G. Rorer's "The great response-style myth". Psychological Bulletin, 66(3), 166-177. https://doi.org/10.1037/h0023709

Schwarz, N., Bless, H., Strack, F., Klumpp, G., Rittenauer-Schatka, H., \& Simons, A. (1991). Ease of Retrieval as Information: Another look at the availability heuristic. Journal of Personality and Social Psychology, 61(2), 195-202. https:// doi.org/10.1037/0022-3514.61.2.195

Shulruf, B., Hattie, J., \& Dixon, R. (2008). Factors affecting responses to Likert questionnaires: introduction of the ImpExp, a new comprehensive model. Social Psychology of Education, 11(1), 59-78. https://doi.org/10.1007/s11218007-9035-x

Soto, C. J., John, O. P., Gosling, S. D., \& Potter, J. (2008). The Developmental Psychometrics of Big Five Self-reports: Acquiescence, factor structure, coherence, and differentiation from ages 10 to 20 . Journal of Personality and Social Psychology, 94(4), 718. https://doi.org/10.1037/00223514.94.4.718

Tourangeau, R., \& Rasinski, K. A. (1988). Cognitive processes underlying context effects in attitude measurement. Psychological Bulletin, 103(3), 299-314. https://doi. org/10.1037/0033-2909.103.3.299
Grazielli Padilha Vieira, Daniely Fernandes Kamazaki Cristiano Weiss, Cristian Zanon e Denise Ruschel Bandeira

Valentini, F. (2017). Influência e controle da aquiescência na análise fatorial. Avaliação Psicológica, 16(2), 116-119. https://doi.org/10.15689/ap.2017.1602.ed

Weijters, B., \& Baumgartner, H. (2012). Misresponse to reversed and negated items in surveys: A review. Journal of Marketing Research, 49(5), 737-747. https://doi.org/10.1509/ jmr.11.0368

Weijters, B., Schillewaert, N., \& Geuens, M. (2008). Assessing response styles across modes of data collection. Journal of the Academy of Marketing Science, 36, 409-422. https:// doi.org/10.1007/s11747-007-0077-6

Wetzel, E., Lüdtke, O., Zettler, I., \& Böhnke, J. R. (2016). The stability of extreme response style and acquiescence over 8 years. Assessment, 23(3), 279-291.https://doi. org/10.1177/1073191115583714

Zanon, C., Brenner, R. E., Baptista, M. N., Vogel, D. L., Rubin, M., Al-Darmaki, F. R., Gonçalves, M., Health, P. J., Liao, H. Y., Mackenzie, C. S., Topkaya, N., Wade, N. G., \& Zlati, A. (2020). Examining the dimensionality, reliability, and invariance of the Depression, Anxiety, and Stress Scale-21 (DASS-21) across eight countries. Assessment, 00 (0), 1-14. https:// doi.org/1073191119887449

Zanon, C., Lessa, J. P. A., \& Dellazzana-Zanon, L. L. (2018). Aquiescência em autorrelatos de personalidade: uma comparação de métodos. Avaliação Psicológica, 17(4), 428-438. https://doi.org/10.15689/ap.2018.1704.3.03

Ziegler, M. (2015). "F*** you, I won't do what you told me!" Response biases as threats to psychological assessment. European Journal of Psychological Assessment, 31(3), 153158. https://doi.org/10.1027/1015-5759/a000292

Zuckerman, M., Knee, C. R., Hodgins, H. S., \& Miyake, K. (1995). Hypothesis confirmation: The joint effect of positive test strategy and acquiescence response set. Journal of Personality and Social Psychology, 68(1), 52-60. https://doi. org/10.1037/0022-3514.68.1.52 